\title{
DIGITALCOMMONS
}

—@WAYNESTATE —

Wayne State University

Physics and Astronomy Faculty Research

Publications

Physics and Astronomy

$1-1-1995$

\section{Asymptotic form of the penetration probability of the quantum harmonic oscillator into the classically forbidden region}

D. E. Atems

Wayne State University

\section{Recommended Citation}

Atems DE \& Wadehra JM. Asymptotic form of the penetration probability of the quantum harmonic oscillator into the classically forbidden region. Am. J. Phys. 1995;63(5):443-6. doi: 10.1119/1.17908

Available at: http://digitalcommons.wayne.edu/phy_astro_frp/83

This Article is brought to you for free and open access by the Physics and Astronomy at DigitalCommons@WayneState. It has been accepted for inclusion in Physics and Astronomy Faculty Research Publications by an authorized administrator of DigitalCommons@WayneState. 
${ }^{\text {aj}}$ On leave from Bar-Ilan University.

${ }^{1}$ L. Fonda, G. C. Ghirardi, and A. Rimini, "Decay theory of unstable quantum systems," Rep. Prog. Phys. 41, 587-631 (1978).

${ }^{2}$ E. Merzbacher, Quantum Mechanics (Wiley, New York, 1970), 2nd ed., pp. 481-486.

${ }^{3}$ V. F. Weisskopf and B. P. Wigner, "Berechnūng der natürlichen Linienbreite auf Grund der Diracsichen Licht theorie," Z. Phys. 63, 54-73 (1930) and V. F. Weisskopf and B. P. Wigner, "Über die naturliche Linienbreite in der Strahlung des harmonischen Oszillâtors," Z. Phys. 65, 18-27 (1930).

${ }^{4}$ C. B. Chiu, E. C. G. Sudarshan, and B. Misra, "Time evolution of unstable quantum states and a resolution of Zeno's paradox," Phys. Rev. D 16, 520-529 (1977).

${ }^{5}$ I. Antoniou, J. Levitan, and L. P. Horwitz, "Time dependence and intrinsic irreversibility of the Pietenpol Model," J. Phys. A 26, 6033-6038 (1993).

${ }^{6}$ A. Peres, "Zeno paradox in quantum theory," Am. J. Phys. 48, 931-932
(1980). It is well known that the decay for very small times in quadratic. Peres notes "that the quadratic time dependence is related to the fact that neighboring quantum states have a stronger resemblance that could be expected classically." See also Ref. 7.

${ }^{7}$ A. Peres, "Unperformed experiments have no results," Am. J. Phys. 46, 745-747 (1978).

${ }^{8}$ L. A. Khalfin, "Contribution to the decay theory of a quasi-stationary state," Sov. Phys. JETP 6(33), 1053-1063 (1958).

${ }^{9}$ N. S. Krylov and V. A. Fock, "On the uncertainty between time and energy," J. Phys. USSR 11, 112-120 (1947).

${ }^{10} \mathrm{~L}$. Stodolsky, "Scattering theofy of resonance mixtures," Phys. Rev. D 1, 2683-2686 (1970).

${ }^{11}$ C. B. Chiu, B. Misra, and E. G. C. Sudarshan, "The time scale for the quantum Zeno paradox and proton decay," Phys. Lett. B 117, 34-40 (1982).

\title{
Asymptotic form of the penetration probability of the quantum harmonic oscillator into the classically forbidden region
}

\author{
D. E. Atems and J. M. Wadehra \\ Department of Physics and Astronomy, Wayne State University, Detroit, Michigan 48202
}

(Received 18 July 1994; accepted 18 October 1994)

\begin{abstract}
The penetration probability of the quantum harmonic oscillator into the classically forbidden region is examined in the limit of large quantum numbers using analytical methods. An expression is derived for the asymptotic form of the penetration probability, and is shown to be in good agreement with an earlier result obtained by numerical methods. The asymptotic form of the probability density in the region between the classical turning points is also presented and found to have a simple physical interpretation. (C) 1995 American Association of Physics Teachers.
\end{abstract}

The harmonic oscillator is one of the most ubiquitous and useful idealizations in physics and physical chemistry, and both its classical and quantum versions have been studied extensively. Surprisingly, however, little seems to be known about the quantum mechanical probability $P(n)$ of finding the oscillator outside the classical turning points when the quantum number $n$ is large, notwithstanding the obvious expectation, based on the correspondence principle, that this probability must somehow tend to zero as the quantum number tends to infinity. A recent article ${ }^{1}$ presented a conjecture, supported by a purely numerical investigation, that this probability has the asymptotic form

$$
P(n)=A(n+1 / 2)^{-1 / 3}-B(n+1 / 2)^{-1}+\cdots
$$

as $n \rightarrow \infty$, with $A \approx 0.133970$ and $B \approx 0.011907$. The main purpose of the present article is to derive an asymptotic formula for this penetration probability using an analytic argument based on the behavior of the harmonic oscillator eigenfunctions in the vicinity of the classical turning points for large quantum numbers. It will be shown that this result is identical in form to Eq. (1). Closed-form expressions will be derived for the coefficients $A$ and $B$, and their values will be found to be quite close to those quoted above. The derivation will also yield the order of the first neglected term; this result will be discussed with the help of a numerical analysis. As a further demonstration of the usefulness of the asymptotic forms of the harmonic oscillator eigenfunctions, a formula for the quantum probability density inside the classical turning points in the large- $n$ limit will be briefly derived and seen to have a simple physical interpretation.

The energy eigenstate of the quantum harmonic oscillator corresponding to the quantum number $n$ has energy $E_{n}=(n+1 / 2) \hbar \omega$ relative to the potential minimum, and the corresponding normalized eigenfunction is given by

$$
\psi_{n}(x)=\left(2^{n} n ! \sqrt{\pi} x_{0}\right)^{-1 / 2} e^{-x^{2} / 2 x_{0}^{2}} H_{n}\left(x / x_{0}\right),
$$

where the $H_{n}$ are the Hermite polynomials, and $x_{0}=\sqrt{\hbar / m \omega}$. The classical turning points occur at

$$
x= \pm \sqrt{2 \nu} x_{0},
$$

where for convenience, both here and later, we put $\nu \equiv n+1 / 2$. The probability of finding the oscillator outside the classical turning points is therefore

$$
\begin{aligned}
P(n) & =2 \int_{\sqrt{2 \nu} x_{0}}^{\infty} d x\left|\psi_{n}(x)\right|^{2} \\
& =2\left(2^{n} n ! \sqrt{\pi} x_{0}\right)^{-1} \int_{\sqrt{2} \nu x_{0}}^{\infty} d x e^{-x^{2} / x_{0}^{2}}\left[H_{n}\left(x / x_{0}\right)\right]^{2}
\end{aligned}
$$

or, changing the variable of integration from $x$ to $y=x / x_{0}$,

$$
P(n)=\frac{1}{2^{n} n !} \frac{2}{\sqrt{\pi}} \int_{\sqrt{2 \nu}}^{\infty} d y e^{-y^{2}}\left[H_{n}(y)\right]^{2}
$$


The integral on the right-hand side of Eq. (2) can be expressed $^{1}$ as a finite sum involving associated Laguerre polynomials and the complementary error function. However, for present purposes (investigating the large- $n$ limit), a more fruitful approach is to directly substitute into the integrand of Eq. (2) a suitable asymptotic representation of the Hermite polynomials. It will be convenient to make use of the following relationship ${ }^{2}$ between the Hermite polynomials $H_{n}(y)$ and the associated Laguerre polynomials $L_{m}^{ \pm 1 / 2}\left(y^{2}\right)$ :

$$
\begin{aligned}
& H_{n}(y) \\
& = \begin{cases}(-1)^{n / 2} 2^{n}\left(\frac{n}{2}\right) ! L_{n / 2}^{-1 / 2}\left(y^{2}\right), & n \text { even } \\
(-1)^{(n-1) / 2} 2^{n}\left(\frac{n-1}{2}\right) ! y L_{(n-1) / 2}^{1 / 2}\left(y^{2}\right), & n \text { odd }\end{cases}
\end{aligned}
$$

Substituting these relations into the integrand of Eq. (2), one obtains

$$
\begin{aligned}
& \frac{1}{2^{n} n !} \frac{2}{\sqrt{\pi}} e^{-y^{2}\left[H_{n}(y)\right]^{2}} \\
& \quad= \begin{cases}2 \frac{\Gamma(\nu / 2+3 / 4)}{\Gamma(\nu / 2+1 / 4)} e^{-y^{2}\left[L_{n / 2}^{-1 / 2}\left(y^{2}\right)\right]^{2},} & n \text { even } \\
2 \frac{\Gamma(\nu / 2+1 / 4)}{\Gamma(\nu / 2+3 / 4)} e^{-y^{2}} y^{2}\left[L_{(n-1) / 2}^{1 / 2}\left(y^{2}\right)\right]^{2}, & n \text { odd }\end{cases}
\end{aligned}
$$

The convenience of this form lies in the fact that there is an asymptotic expansion, involving powers of $\nu^{3}$ of the $L_{m}^{\alpha}\left(y^{2}\right)$ in the vicinity of the turning points at $y^{2}=2 \nu$. Defining a new variable $\xi$ by the relation $y^{2}=2 \nu\left(1+\nu^{-2 / 3} \xi\right)$, this expansion can be expressed as

$$
\begin{aligned}
e^{-y^{2} / 2} L_{m}^{\alpha}\left(y^{2}\right)= & (-1)^{m} 2^{-\alpha} \nu^{-1 / 3}\left\{\operatorname{Ai}(\xi)-\frac{1}{2}\left[\frac{2}{5} \xi^{2} \mathrm{Ai}^{\prime}(\xi)\right.\right. \\
& \left.\left.+\left(\frac{3}{5}+\alpha\right) \xi \operatorname{Ai}(\xi)\right] \nu^{-2 / 3}+O\left(\nu^{-4 / 3}\right)\right\}
\end{aligned}
$$

where $m=n / 2$ or $(n-1) / 2$ according as $n$ is even or odd, $\mathrm{Ai}(\xi)$ is the Airy function, defined ${ }^{4}$ in terms of the modified Bessel function $K_{1 / 3}(z)$ as

$$
\operatorname{Ai}(\xi)=\frac{1}{\pi} \sqrt{\frac{\xi}{3}} K_{1 / 3}\left(\frac{2}{3} \xi^{3 / 2}\right)
$$

and the prime denotes differentiation with respect to the argument. Substitution of Eq. (5) into Eq. (4) then leads, with the help of the asymptotic form of the ratio of two Gamma functions, ${ }^{5}$

$$
\frac{\Gamma(z+a)}{\Gamma(z+b)}=z^{a-b}\left[1+\frac{1}{2 z}(a-b)(a+b-1)+O\left(z^{-2}\right)\right],
$$

to the following expansion of the integrand in Eq. (2):

$$
\begin{aligned}
\frac{1}{2^{n} n !} & \frac{2}{\sqrt{\pi}} e^{-y^{2}}\left[H_{n}(y)\right]^{2} \\
= & 2(2 \nu)^{1 / 2}\left\{\operatorname{Ai}^{2}(\xi) \nu^{-2 / 3}-\left[\frac{2}{5} \xi^{2} \operatorname{Ai}^{\prime}(\xi) A i(\xi)\right.\right. \\
& \left.\left.+\frac{1}{10} \xi \operatorname{Ai}^{2}(\xi)\right] \nu^{-4 / 3}+O\left(\nu^{-2}\right)\right\},
\end{aligned}
$$

which holds for both even and odd $n$. It is readily verified numerically that for sufficiently large $n$, the approximation afforded by the two explicitly given terms of this expansion is extremely close to the exact integrand. For $n \geq 50$, the two quantities differ by less than $1 \%$ over the range of $x$ responsible for over $99 \%$ of the contribution to the integral itself.

If Eq. (7) is substituted into Eq. (2), then after changing the integration variable from $y$ to $\xi$, the penetration probability becomes

$$
\begin{aligned}
P(n)= & 2 \int_{0}^{\infty} d \xi\left[1-\frac{1}{2} \nu^{-2 / 3} \xi+O\left(\nu^{-4 / 3}\right)\right] \\
& \times\left\{\operatorname{Ai}^{2}(\xi) \nu^{-1 / 3}-\left[\frac{2}{5} \xi^{2} \operatorname{Ai}^{\prime}(\xi) \operatorname{Ai}(\xi)\right.\right. \\
& \left.\left.+\frac{1}{10} \xi \operatorname{Ai}^{2}(\xi)\right] \nu^{-1}+O\left(\nu^{-5 / 3}\right)\right\}
\end{aligned}
$$

where the first factor in parentheses under the integral sign results from expressing $d y$ as a function of $\xi$ and expanding in powers of $\nu^{-2 / 3} \xi^{6}$ Termwise integration of Eq. (8) then leads to

$$
P(n) \approx 2 I_{1} \nu^{-1 / 3}-\left(\frac{4}{5} I_{3}+\frac{6}{5} I_{2}\right) \nu^{-1}+O\left(\nu^{-5 / 3}\right)
$$

with

$$
\begin{aligned}
& I_{1}=\int_{0}^{\infty} d \xi \operatorname{Ai}^{2}(\xi)=\frac{3^{1 / 3}}{4 \pi^{2}}\left[\Gamma\left(\frac{2}{3}\right)\right]^{2}, \\
& I_{2}=\int_{0}^{\infty} d \xi \xi \operatorname{Ai}^{2}(\xi)=\frac{1}{6 \pi \sqrt{3}}, \\
& I_{3}=\int_{0}^{\infty} d \xi \xi^{2} \operatorname{Ai}^{\prime}(\xi) \operatorname{Ai}(\xi)=-\int_{0}^{\infty} d \xi \xi \operatorname{Ai}^{2}(\xi)=-I_{2},
\end{aligned}
$$

where the second equality on the last line follows by partial integration. The integrals $I_{1}$ and $I_{2}$ are easily evaluated using the standard form ${ }^{7}$

$$
\begin{aligned}
\int_{0}^{\infty} d x x^{\alpha-1} K_{\mu}^{2}(x)= & \frac{2^{\alpha-3}}{\Gamma(\alpha)} \Gamma\left(\frac{\alpha}{2}+\mu\right) \Gamma\left(\frac{\alpha}{2}-\mu\right) \\
& \times\left[\Gamma\left(\frac{\alpha}{2}\right)\right]^{2}
\end{aligned}
$$

and the definition (6) of $\operatorname{Ai}(\xi)$. The asymptotic form of the penetration probability is thus found to be approximately

$$
\begin{aligned}
P(n) \approx & A(n+1 / 2)^{-1 / 3}-B(n+1 / 2)^{-1} \\
& +O\left[(n+1 / 2)^{-5 / 3}\right]
\end{aligned}
$$

with 


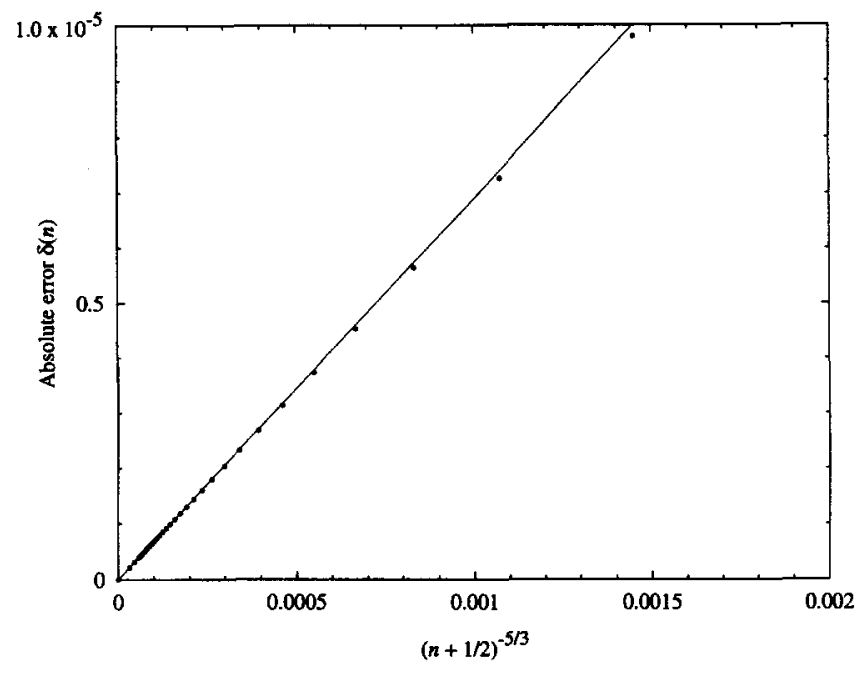

Fig. 1. The absolute error $\delta(n)$ for $n=50,60,70, \ldots, 350,400,500$, and the straight line passing through the origin and the point corresponding to $n=500$.

$$
\begin{aligned}
& A=\frac{3^{1 / 3}}{2 \pi^{2}}\left[\Gamma\left(\frac{2}{3}\right)\right]^{2} \approx 0.133975, \\
& B=\frac{1}{15 \pi \sqrt{3}} \approx 0.012252,
\end{aligned}
$$

in good agreement with the values quoted in the first paragraph of this article, which were obtained by numerical methods. ${ }^{1}$

Note that the $O$ term in Eq. (9) comes directly from the asymptotic expansion (7) of the integrand in Eq. (2). It is of interest to examine the absolute error in the first two terms of Eq. (9). Denote this quantity as $\delta(n)$; i.e., define

$$
\delta(n) \equiv P(n)-A(n+1 / 2)^{-1 / 3}+B(n+1 / 2)^{-1} .
$$

In Fig. 1 the absolute error is plotted against $(n+1 / 2)^{-5 / 3}$ for $n=50,60,70, \ldots, 350,400,500$ using the coefficients $A$ and $B$ given by Eq. (10). The values of $P(n)$ were obtained by numerical integration of the right-hand side of Eq. (4) with respect to $y^{2}$, using Simpson's rule, on a grid of step size 0.01 , in $\mathrm{REAL}^{*} 8$ precision on a SGI Iris Indigo computer. The associated Laguerre polynomials were generated using a standard recurrence relation. ${ }^{8}$ The solid line in the figure is defined by connecting the last point in the set (i.e., $n=500$ ) to the origin. It is noteworthy that for $n \geq 100$, all of the values of $\delta(n)$ lie very nearly on this line, a fact which is consistent with the $O$ term in Eq. (9).

It is also interesting to consider the probability density in the state $\psi_{n}$ inside the classical turning points. In elementary textbooks on quantum mechanics ${ }^{9}$ one often sees plots like the one in Fig. 2, showing, for some large value of $\boldsymbol{n}$ (in this case $n=50$ ), the probability density as calculated from quantum mechanics, compared with the corresponding classical probability density

$$
P_{\mathrm{cl}}(x)=\pi^{-1}\left(2 \nu x_{0}^{2}-x^{2}\right)^{-1 / 2}
$$

for a harmonic oscillator of the same energy. ${ }^{10}$ Intuitively one sees that the "envelope" of the quantum curve follows the classical curve more and more closely as $n$ increases. This can be seen analytically as well; yet this fact is not shown in any textbook of which the authors are aware.

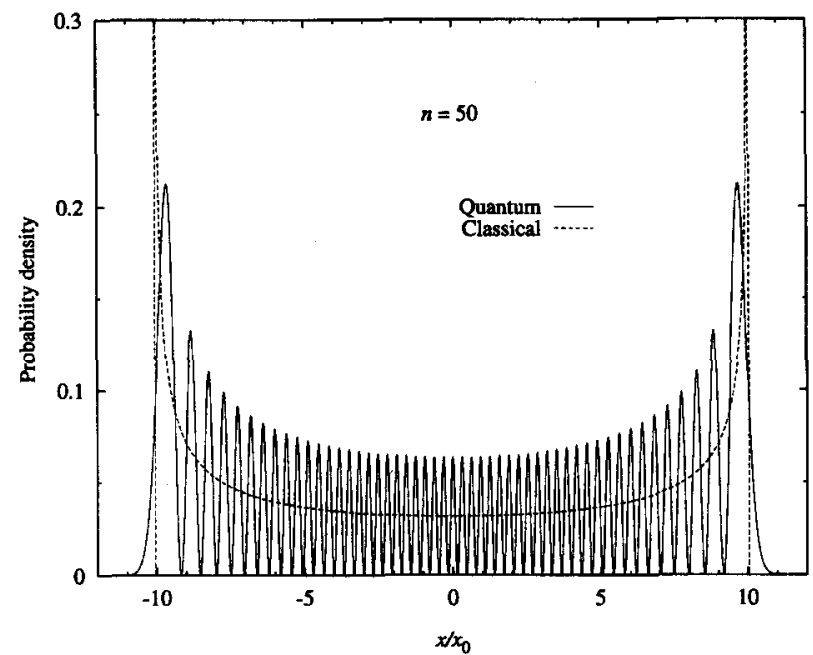

Fig. 2. The dimensionless probability density $x_{0}\left|\psi_{n}(x)\right|^{2}$ of a quantum harmonic oscillator for $n=50$, and the corresponding probability density $\pi^{-1}\left(2 \nu-x^{2} / x_{0}^{2}\right)^{-1 / 2}$ for a classical oscillator of the same energy.

The asymptotic series [Eq. (5)] is one of a collection of expansions, ${ }^{3}$ each of which describes the behavior of the $L_{m}^{\alpha}\left(y^{2}\right)$ for large order in a different subdomain of the argument. For the subdomain corresponding to the region inside the classical turning points, i.e., $0<y^{2}<2 \nu$, one has

$$
\begin{aligned}
e^{-y^{2} / 2} L_{m}^{\alpha}\left(y^{2}\right)= & 2(-1)^{m}(2 \cos \theta)^{-\alpha}(2 \pi \nu \sin 2 \theta)^{-1 / 2} \\
& \times\left\{\sin \left[\nu\left(\theta-\frac{1}{2} \sin 2 \theta\right)+\frac{\pi}{4}\right]+O\left(\nu^{-1}\right)\right\},
\end{aligned}
$$

where $y=\sqrt{2 \nu} \cos \theta$ and, as before, $\nu=n+1 / 2$ and $m$ is $n / 2$ or $(n-1) / 2$ according as $n$ is even or odd, respectively. It is then readily shown with the help of Eq. (4), using an argument completely analogous to the one leading to Eq. (7), that

$$
\begin{aligned}
\left|\psi_{n}(x)\right|^{2}= & \frac{1}{2^{n} n ! \sqrt{\pi} x_{0}} e^{-x^{2} / x_{0}^{2}}\left[H_{n}\left(x / x_{0}\right)\right]^{2} \\
\approx & \frac{1}{\pi x_{0}(2 \nu)^{1 / 2} \sin \theta} \\
& \times\left\{2 \sin ^{2}\left[\nu\left(\theta-\frac{1}{2} \sin 2 \theta\right)+\frac{\pi}{4}\right]+O\left(\nu^{-1}\right)\right\} \\
= & P_{\mathrm{cl}}(x)\left\{2 \sin ^{2}\left[\nu\left(\theta-\frac{1}{2} \sin 2 \theta\right)+\frac{\pi}{4}\right]+O\left(\nu^{-1}\right)\right\}
\end{aligned}
$$

as $n \rightarrow \infty$. Thus to leading order, the quantum probability density inside the classical turning points is indeed, for large quantum numbers, a rapidly oscillating function modulated by a more slowly varying envelope consisting of its classical counterpart.

In conclusion, we have analytically derived an asymptotic formula for the penetration probability of the quantum harmonic oscillator into the classically forbidden region for large quantum numbers, and seen that it is in reasonably good agreement with an asymptotic formula obtained previously ${ }^{1}$ by numerical methods. We have also used a nu- 
merical calculation to support the derived order of the error in the first two terms of this formula. We cannot, at present, supply a meaningful physical interpretation for this asymptotic form. ${ }^{11}$ Finally, we have shown that the leading-order term in the asymptotic expansion, for large quantum numbers, of the quantum probability density inside the classical turning points has a form which is readily understood physically in light of the correspondence principle.

\section{ACKNOWLEDGMENT}

Support of this work by AFOSR (Grant No. F49620-92-J0027 ) is gratefully acknowledged.

${ }^{1}$ J. J. Diamond, "Classically forbidden behavior of the quantum harmonic oscillator for large quantum numbers," Am. J. Phys. 60, 912-916 (1992). ${ }^{2}$ Handbook of Mathematical Functions, edited by M. Abramowitz and I. A. Stegun (Dover, New York, 1965), p. 779.

${ }^{3}$ A. Erdelyi, W. Magnus, F. Oberhettinger, and F. G. Tricomi, Higher Transcendental Functions (McGraw-Hill, New York, 1953), Vol. 2, p. 200.

${ }^{4}$ See Ref. 2, p. 447. In Ref. 3, the asymptotic expansion [Eq. (5)] is expressed in terms of a different but closely related "Airy function" $A(t)=3^{-1 / 3} \pi \mathrm{Ai}\left(-t / 3^{1 / 3}\right)$. $\mathrm{Ai}(z)$ seems to be more commonly used.
${ }^{5}$ Reference 2, p. 257.

${ }^{6}$ Here the integrand decays so rapidly that virtually the entire contribution to the integral in Eq. (8) lies in the interval $0 \leqslant \xi \leqslant 4$ for all values of $\nu$. Thus for $\nu$ sufficiently large, the series expansion for $\left(1+\nu^{-2 / 3} \xi\right)^{-1 / 2}$ may justifiably be regarded, for all practical purposes, as convergent throughout the domain of integration.

${ }^{7}$ I. S. Gradshteyn and I. M. Ryzhik, Table of Integrals, Series, and Products, corrected and enlarged edition (Academic, New York, 1980), p. 693. The form used here is a special case of Eq. (6.576.4).

${ }^{8}$ Reference 2, p. 782.

${ }^{9}$ See, for example, L. I. Schiff, Quantum Mechanics, 3rd ed. (McGraw-Hill, New York, 1968), p. 74; also L. Pauling and E. B. Wilson, Jr., Introduction to Quantum Mechanics (McGraw-Hill, New York, 1935), p. 76.

${ }^{10}$ The classical probability density [Eq. (12)] is easily derived by considering the fraction of time the oscillator spends in the interval between $x$ and $x+d x$; that fraction is just $P_{\mathrm{cl}}(x) d x$.

${ }^{11}$ Because the leading term in Eq. (9) falls off so slowly as the quantum number increases, it is tempting to infer (as in Ref. 1) that significantly nonclassical behavior persists even when $n$ is very large. However, one should keep in mind that the probability density in the classically forbidden region decays more rapidly with increasing $n$, because of the increasing steepness of the barrier at the classical turning point. Thus, although the total penetration probability remains appreciable even for very large $n$, most of the contribution to this probability comes increasingly from the immediate vicinity of the turning points.

\title{
Quantum hydrodynamics and virial theorems
}

\author{
Peter J. Price \\ IBM T. J. Watson Research Center, Yorktown Heights, New York 10598
}

(Received 18 July 1994; accepted 17 October 1994)

This paper elucidates how many-particle quantum hydrodynamic equations underlie the virial formula, including its surface pressure term. It shows the relation of the field variables to the particle Wigner function, proposes a many-particle "liquid" model to approximate these variables, and discusses its application to electrons in semiconductor nanostructures. (C) 1995 American Association of Physics Teachers.

Virial theorems, a recurring topic in this journal, are instructive and useful. The recent paper by Muga ${ }^{1}$ discusses time versus ensemble averages in a many-particle version. It additionally draws attention to the seemingly anomalous surface term that appears when $(\psi|[\hat{A}, \hat{B}]| \psi)$, the Schrödinger evaluation of a commutator, is integrated over a finite enclosing space with the usual "confinement" boundary conditions for the wave function $\psi$. In particular, when $\hat{A}$ and $\hat{B}$ are, respectively, the particle Hamiltonian and the symmetrized product of position and momentum vectors, as in the common derivation of the virial theorem by means of quantum operator algebra, this anomalous contribution from the bounding surface is then the "missing" pressure term of the classical formula. Its mathematical origin is a divergence between formal representation theory, according to which the expectation of any such operator commutator is zero, and the explicit evaluation in terms of a Schrödinger wave function..$^{2-4}$ The present communication outlines a correct derivation of the virial formula in terms of "hydrodynamic" field equations (including a time-dependence term), and con- siders the adaptation of these underlying equations to the analysis of electron flow in semiconductor structures such as nanostructures.

The Hamiltonian assumed is the many-particle

$$
H=\left(\hbar^{2} / 2 M\right) \Sigma_{n} p_{n}^{2}+U\left(\mathbf{r}_{1} \cdots \mathbf{r}_{N}\right),
$$

where the sum is over the $N$ particles. (The masses are taken to be all equal, as will be the case for identical particles.) It may (but need not) be assumed that the potential is given by $U=U_{\mathrm{I}}+U_{\text {II }}$, the sum of a single-particle term $U_{\mathrm{I}}=\Sigma_{n} W_{1}\left(\mathbf{r}_{n}\right)$ and a pair term $U_{\mathrm{II}}=\Sigma_{n<m} W_{2}\left(\mathbf{r}_{n m}\right)$. For the state with wave function $\psi\left(\mathbf{r}_{1} \cdots \mathbf{r}_{N}\right)$, the single-particle density may be written

$$
w(\mathbf{r})=\Sigma_{n} I_{n} \psi^{*} \psi
$$

and the momentum density of the particle flow similarly

$$
\mathbf{J}(\mathbf{r})=\frac{1}{2} i \hbar \Sigma_{n} I_{n}\left(\psi \nabla_{n} \psi^{*}-\psi^{*} \nabla_{n} \psi\right),
$$

where $I_{n}$ means integration $\int d^{3} \mathbf{r}_{1} \cdots \int d^{3} \mathbf{r}_{N}$ over the finite domain $(N-1)$ times, for all of the $\mathbf{r}_{m}$ except $\mathbf{r}_{n}$, then dropping the subscript of $\mathbf{r}_{n}$. From the Schrödinger equation $i \hbar \partial \psi / \partial t$ 